\title{
The overexpression of the trypanosomatid-exclusive TcRBP19 RNA- binding protein affects cellular infection by Trypanosoma cruzi
}

\author{
Leticia Pérez-Díaz', Alejandro Correa², Mariana P Moretão², \\ Samuel Goldenberg², Bruno Dallagiovanna ${ }^{2 /+}$, Beatriz Garat ${ }^{1}$ \\ ${ }^{1}$ Laboratorio de Interacciones Moleculares, Facultad de Ciencias, Iguá, Montevideo, Uruguay \\ ${ }^{2}$ Instituto Carlos Chagas-Fiocruz, Curitiba, PR, Brasil
}

To characterise the trypanosomatid-exclusive RNA-binding protein TcRBP19, we analysed the phenotypic changes caused by its overexpression. Although no evident changes were observed when TcRBP19 was ectopically expressed in epimastigotes, the metacyclogenesis process was affected. Notably, TcRBP19 overexpression also led to a decrease in the number of infected mammalian cells. These findings suggest that TcRBP19 may be involved in the life cycle progression of the Trypanosoma cruzi parasite.

Key words: differentiation - metacyclogenesis - amastigogenesis

The protozoan parasite Trypanosoma cruzi is the aetiologic agent of Chagas' disease, which is one of the most significant human health problems in South and Central America (Lima et al. 2010). Only two therapeutic options for Chagas' disease, benznidazole and nifurtimox, have been available for over 40 years. These drugs are primarily effective during the early-acute phase; in chronically infected patients, they are considered less effective and their use can even lead to resistance. The severe side effects associated with benznidazole and nifurtimox and the absence of paediatric formulations (a serious issue because the majority of acute Chagas disease patients are children) underscore the drawbacks of these drugs (Canavaci et al. 2010). The characterisation of T. cruzi-specific molecules or processes could provide a basis for the development of novel tools for appropriate disease treatments.

The T. cruzi life cycle comprises at least four stages: in the triatomine insect, the epimastigote differentiates into the metacyclic trypomastigote, which can invade a mammalian host and transform into the replicative intracellular amastigote. The amastigote stage ultimately differentiates into the trypomastigote bloodstream form, which destroys the cell and propagates the infection by invading more host cells (Lima et al. 2010). The mechanisms that regulate these differentiation steps remain poorly understood (Martinez-Calvillo et al. 2010). Because there appears to be virtually no regulation of transcription initiation for protein-coding genes in T. cruzi, the regulation of their expression is thought to depend

Financial support: FCE (ANII), CSIC, CNPq Prosul Program LP-D is supported by fellowships from CSIC, PEDECIBA, AMSUDPasteur and RTPD, BD from FIOCRUZ-CNPq, SG from CNPq and $\mathrm{BG}$ from PV-CNPq.

+Corresponding author: brunod@tecpar.br

Received 14 August 2012

Accepted 31 October 2012 mainly on post-transcriptional processes (Clayton \& Shapira 2007, Haile \& Papadopoulou 2007). The mature mRNAs are derived from polycistronic transcripts that are produced at relatively constant levels (except for occasional genomic copy amplification) and are regulated at the level of degradation, localisation or translation via untranslated region signals and RNA-binding proteins (Kramer \& Carrington 2011). Several of the $T$. cruzi regulatory proteins have been identified; however, the mechanisms governing the developmental differentiation process of the parasite remain largely unknown (Araujo \& Teixeira 2011).

We previously identified a $17-\mathrm{kDa}$ RNA-binding protein, TcRBP19, that is exclusive to trypanosomatids and is differentially expressed during the life cycle of $T$. cruzi, being detected only in the amastigote stage (PerezDiaz et al. 2007). The RNA-binding domain of TcRBP19 belongs to the RNA-recognition motif family (RRM), which is widely represented in proteins involved in posttranscriptional events, such as RNA processing, transport, translation, degradation and stabilisation (Dreyfuss et al. 2002). In the T. cruzi genome, 77 open reading frames that code for putative RRM-containing proteins have been identified (De Gaudenzi et al. 2005); however, with few exceptions, the expression and functional roles of these proteins remain to be characterised (D'Orso \& Frasch 2002, De Gaudenzi et al. 2003, Noe et al. 2008, Cassola \& Frasch 2009). The low levels of TcRBP19 expression, together with its ability to discriminate between RNA targets, led us to propose the involvement of this protein in specific regulatory steps of gene expression (Perez-Diaz et al. 2007).

Because no functional RNA interference mechanism has been demonstrated in T. cruzi (da Rocha et al. 2004) and only a few cases of gene knockdown have been reported, overexpression constitutes the major approach to address functional roles in this parasite. Therefore, we studied the phenotypic changes resulting from TcRBP19 overexpression during the $T$. cruzi life cycle. In this study, we present a comparative analysis of the in vitro 
differentiation of epimastigotes of T. cruzi Dm28 clone into metacyclic trypomastigotes and amastigotes using pTEX-TcRBP19 and control pTEX transfectants.

The transfectant epimastigotes were maintained at $28^{\circ} \mathrm{C}$ in a liver infusion tryptose medium supplemented with $10 \%$ heat-inactivated foetal bovine serum and 250 $\mu \mathrm{g} / \mathrm{mL} \mathrm{G-418} \mathrm{to} \mathrm{provide} \mathrm{selective} \mathrm{pressure} \mathrm{to} \mathrm{maintain}$ a low plasmid copy number in the transfected population. Polymerase chain reaction (PCR) with vector primers and western blotting with a specific anti-TcRBP19 antibody (Perez-Diaz et al. 2007) were done for confirmation of transfectant population. An average overexpression level of approximately $1 \mathrm{ng}$ of TcRBP19 per $\mu \mathrm{g}$ of total protein extract was estimated using western
A

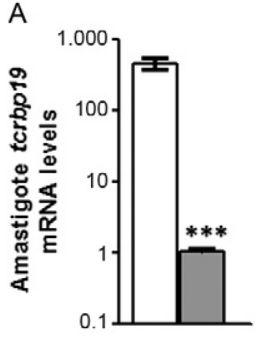

B

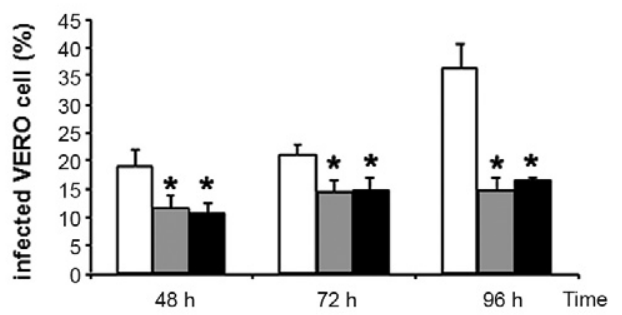

d
C a

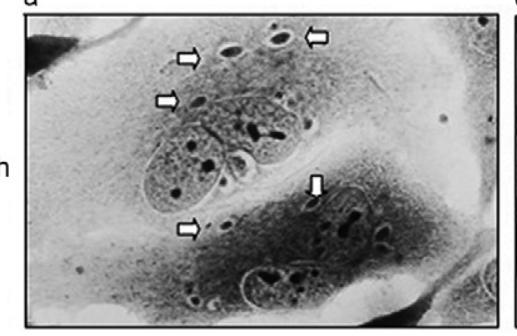

b

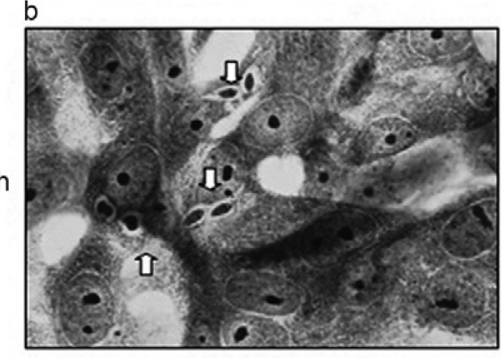

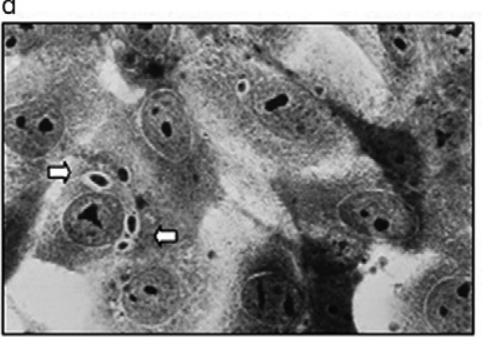

e
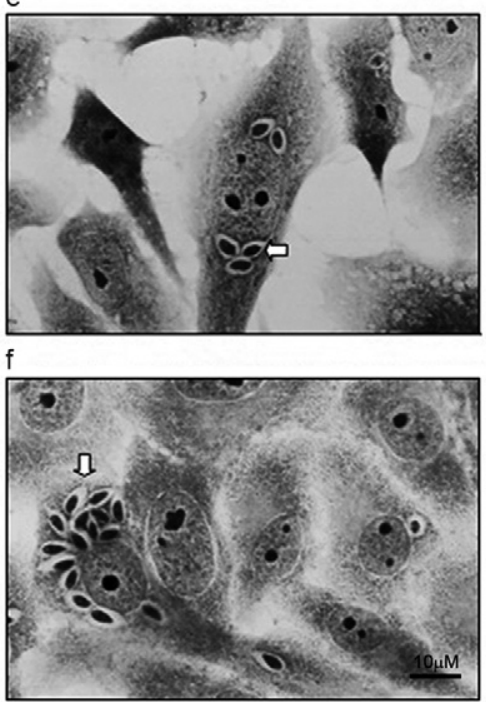

Fig. 1: infectivity of TcRBP19 overexpressing parasites in VERO cells (A). Amastigote tcrbp19 mRNA quantification by quantitative reverse transcription-polymerase chain reaction. tcrbp19 gene specific primers (Tc19fw 5'-AGGCATTCAGCGTTACAAGAAC-3' and Tc19rev 5'CGATTTGCCAAGAAGTTTGTG-3') were used to analyze overexpression in cellular amastigotes relative to gapdh used as a housekeeping mRNA (lpgapdh1 5'-CGACAACGAGTGGGGATACT-3' and lpgapdh2 5'-CTACAACCTTGCCGAACGAT-3' primers). Each point corresponds to the average obtained from quadruplicates corresponding to the two independent plasmid transfection (pTEX-TcRBP19 A and B) and to the control (pTEX). Results are expressed as means \pm standard deviation. Normality and homogeneity were evaluated by analysis of variance (ANOVA). t-test was used to determine the statistical significance $\left(^{*}\right)$ difference $\left.{ }^{* *} \mathrm{p}<0.0001\right)$; B: percentage of infected VERO cells using TcRBP19 overexpressing parasites. The two independent transfectant are indicated in gray (pTEX-TcRBP19 A) and black (pTEX-TcRBP19 B), respectively, and the pTEX transfectant control in white. Assays were done in quadruplicates and 300 cells were counted in each experiment. Normality and homogeneity data were evaluated by ANOVA. Tukey's test was used to determine the statistical significance. $\left({ }^{*}\right)$ difference $(* p<$ 0.05); C: representative images of infected VERO cells. Monolayers were infected with pTEX (a-c) and pTEX-TcRBP19-A (d-f) transfectants at different incubation times. VERO cells were infected with metacyclic forms of the transfectant parasites (pTEX or pTEX-TcRBP19) (1:10). After $24 \mathrm{~h}$ of infection, the infected monolayers were cultured with daily medium changes. Cultures were followed by optical microscopy. Arrows point to the intracellular forms of the transfectants. Original magnification: 1.000X. 
blotting. Metacyclic trypomastigotes were prepared and purified in diethylaminoethyl cellulose columns (Contreras et al. 1985) and were then used to infect VERO or HeLa cells in a 10:1 parasite:mammalian cell ratio. The trypomastigote motility was checked using optical microscopy prior to infection. The infected monolayers were cultured in RPMI medium (Sigma) at $37^{\circ} \mathrm{C}$ without shaking in a $5 \% \mathrm{CO}_{2}$ atmosphere for four days, with a daily medium change beginning $24 \mathrm{~h}$ after infection. Immunoflourescent assay was used to confirm TcRBP19 overexpression during the different parasite life cycle stages. The results of quantitative reverse transcriptionPCR revealed a 475 -fold increase in tcrbp 19 mRNA in the TcRBP19-overexpressing amastigotes compared with the pTEX control parasites (Fig. 1A). In a previous study, we found no phenotypic differences between epimastigotes ectopically overexpressing TcRBP19 and control transfectants carrying the empty vector (PerezDiaz et al. 2007).

Regarding metacyclogenesis, we found that the ectopic overexpression of TcRBP19 produced a reduction of the metacyclic stage yield. Indeed, although $40 \%$ of the cells transfected with the pTEX control vector differentiated into metacyclic trypomastigotes, only $26-28 \%$ of the pTEX-TcRBP19 transfectants underwent a similar differentiation (Table).

In contrast, there were no significant differences between the pTEX-TcRBP19 transfectants and the controls in terms of the mean numbers of intracellular amastigote parasites per infected cell (Table). However, at all the analysed time points, the percentage of infected VERO cells was significantly lower for the pTEX-TcRBP19 transfectants compared with the pTEX control-transfected parasites (Fig. 1B). Therefore, TcRBP19 overexpression at the intracellular stage of $T$. cruzi life cycle, in which the protein is usually present, neither introduced obvious phenotypic changes nor affected the replication ability. However, the cellular infectivity of the trypomastigotes that ectopically overexpressed TcRBP19 was reduced. Fig. 1C shows representative images of the infectivity of the control pTEX (a-c) and pTEX-TcRBP19 (d-f) transfectants at different incubation times. Consistent results were obtained for two independent pTEXTcRBP19 transfections.

HeLa cells were also infected with these parasites. Whereas $37 \%$ of the HeLa cells became infected using the pTEX transfectants, only $8 \%$ were infected when the
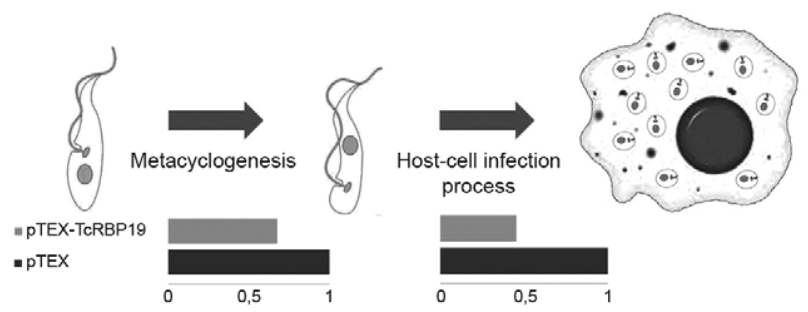

Fig. 2: graphical representation of the effects of TcRBP19 overexpression through Trypanosoma cruzi differentiation.
pTEX-TcRBP19 transfectants were used. Thus, similar reductions in the infection efficiency of parasites overexpressing TcRBP19 were observed in two different host cell lines. It is worth mentioning that we previously used an identical approach to characterise the RNA-binding protein TcPuf6. In that case, no effects on either metacyclogenesis or amastigogenesis were observed (Dallagiovanna et al. 2008). The data presented here suggest that the ectopic overexpression of TcRBP19 affects the successful differentiation of epimastigotes into the infective metacyclic form (Fig. 2).

The reduced yield of metacyclic pTEX-TcRBP19 trypomastigotes compared with the pTEX-transfected parasite yield led us to hypothesise that either the ability to interpret and/or respond to the mechanisms triggered by nutritional stress signals or the differentiation process itself may be affected by the ectopic expression of TcRBP19. Modifying the levels of this RNA-binding protein may produce imbalances in the gene regulatory networks that facilitate the life cycle progression of the T. cruzi parasite.

Moreover, the reduction in the number of infected mammalian cells caused by TcRBP19 overexpression constitutes further evidence of a regulatory role for this protein. The identification of target genes whose expression is regulated by TcRBP19 and the roles of these genes in the mechanisms of infection, cell adherence and/or invasion require further research.

The data presented here indicate that the control of TcRBP19 expression is required to achieve completely efficient metacyclogenesis and productive infection.

These findings, together with the observation that TcRBP19 orthologues are present only in trypanosomatids (Perez-Diaz et al. 2007), support the importance of pursuing further the analysis and characterisation of this RNA-binding protein as a potential tool in the fight against trypanosomatid illnesses. The identification and characterisation of factors involved in the regulation of differentiation and infection could also enhance our understanding of the parasite-host interaction and distinctive molecular processes.

TABLE

Effect of TcRBP19 overexpression on life cycle development

\begin{tabular}{lccc}
\hline & & \multicolumn{3}{c}{$\begin{array}{c}\text { Means of } \\
\text { intracellular }\end{array}$} \\
Time from induction & $\begin{array}{c}\text { Metacycliclic/total } \\
\text { amastigotes/cell } \\
\text { a }\end{array}$ & $48 \mathrm{~h}$ & $96 \mathrm{~h}$ \\
\hline pTEX & 40 & 1.32 & 18.52 \\
pTEX-TcRBP19-A & 26 & 1.27 & 16.35 \\
pTEX-TcRBP19-B & 28.8 & 1.14 & 17.80
\end{tabular}

$a$ : statistical significance difference (chi-square test, $\mathrm{p}<$ $0.01)$; A, B: two independent transfections. Developmental stages of Trypanosoma cruzi transfected with the listed vectors are indicated. 


\section{REFERENCES}

Araujo PR, Teixeira SM 2011. Regulatory elements involved in the post-transcriptional control of stage-specific gene expression in Trypanosoma cruzi - A Review. Mem Inst Oswaldo Cruz 106: 257-266.

Canavaci AM, Bustamante JM, Padilla AM, Perez Brandan CM, Simpson LJ, Xu D, Boehlke CL, Tarleton RL 2010. In vitro and in vivo high-throughput assays for the testing of anti-Trypanosoma cruzi compounds. PLoS Negl Trop Dis 4: e740.

Cassola A, Frasch AC 2009. An RNA recognition motif mediates the nucleocytoplasmic transport of a trypanosome RNA-binding protein. J Biol Chem 284: 35015-35028.

Clayton C, Shapira M 2007. Post-transcriptional regulation of gene expression in trypanosomes and leishmanias. Mol Biochem Parasitol 156: 93-101.

Contreras VT, Salles JM, Thomas N, Morel CM, Goldenberg S 1985. In vitro differentiation of Trypanosoma cruzi under chemically defined conditions. Mol Biochem Parasitol 16: 315-327.

Dallagiovanna B, Correa A, Probst CM, Holetz F, Smircich P, de Aguiar AM, Mansur F, da Silva CV, Mortara RA, Garat B, Buck GA, Goldenberg S, Krieger MA 2008. Functional genomic characterization of mRNAs associated with TcPUF6, a pumilio-like protein from Trypanosoma cruzi. J Biol Chem 283: 8266-8273.

da Rocha WD, Otsu K, Teixeira SM, Donelson JE 2004. Tests of cytoplasmic RNA interference (RNAi) and construction of a tetracycline-inducible $\mathrm{T} 7$ promoter system in Trypanosoma cruzi. Mol Biochem Parasitol 133: 175-186.

De Gaudenzi J, Frasch AC, Clayton C 2005. RNA-Binding domain proteins in kinetoplastids: a comparative analysis. Eukaryot Cell 4: $2106-2114$.
De Gaudenzi JG, D’Orso I, Frasch AC 2003. RNA recognition motiftype RNA-binding proteins in Trypanosoma cruzi form a family involved in the interaction with specific transcripts in vivo. J Biol Chem 278: 18884-18894.

D’Orso I, Frasch AC 2002. TcUBP-1, an mRNA destabilizing factor from trypanosomes, homodimerizes and interacts with novel AU-rich element and poly(A)-binding proteins forming a ribonucleoprotein complex. J Biol Chem 277: 50520-50528.

Dreyfuss G, Kim VN, Kataoka N 2002. Messenger-RNA-binding proteins and the messages they carry. Nat Rev Mol Cell Biol 3: 195-205.

Haile S, Papadopoulou B 2007. Developmental regulation of gene expression in trypanosomatid parasitic protozoa. Curr Opin Microbiol 10: 569-577.

Kramer S, Carrington M 2011. Trans-acting proteins regulating mRNA maturation, stability and translation in trypanosomatids. Trends Parasitol 27: 23-30.

Lima FM, Oliveira P, Mortara RA, Silveira JF, Bahia D 2010. The challenge of Chagas' disease: has the human pathogen, Trypanosoma cruzi, learned how to modulate signaling events to subvert host cells? N Biotechnol 27: 837.

Martinez-Calvillo S, Vizuet-de-Rueda JC, Florencio-Martinez LE, Manning-Cela RG, Figueroa-Angulo EE 2010. Gene expression in trypanosomatid parasites. J Biomed Biotechnol: 525241.

Noe G, De Gaudenzi JG, Frasch AC 2008. Functionally related transcripts have common RNA motifs for specific RNA-binding proteins in trypanosomes. BMC Mol Biol 9: 107.

Perez-Diaz L, Duhagon MA, Smircich P, Sotelo-Silveira J, Robello C, Krieger MA, Goldenberg S, Williams N, Dallagiovanna B, Garat B 2007. Trypanosoma cruzi: molecular characterization of an RNA binding protein differentially expressed in the parasite life cycle. Exp Parasitol 117: 99-105. 